УДК $517.53+517.58$

T. H. Nguyen, A. Vishnyakova

\title{
ON ENTIRE FUNCTIONS FROM THE LAGUERRE-PÓLYA I CLASS WITH NON-MONOTONIC SECOND QUOTIENTS OF TAYLOR COEFFICIENTS
}

\begin{abstract}
T. H. Nguyen, A. Vishnyakova. On entire functions from the Laguerre-Pólya I class with nonmonotonic second quotients of Taylor coefficients, Mat. Stud. 56 (2021), 149-161.

For an entire function $f(z)=\sum_{k=0}^{\infty} a_{k} z^{k}, a_{k}>0$, we define its second quotients of Taylor coefficients as $q_{k}(f):=\frac{a_{k-1}^{2}}{a_{k-2} a_{k}}, k \geq 2$. In the present paper, we study entire functions of order zero with non-monotonic second quotients of Taylor coefficients. We consider those entire functions for which the even-indexed quotients are all equal and the odd-indexed ones are all equal: $q_{2 k}=a>1$ and $q_{2 k+1}=b>1$ for all $k \in \mathbb{N}$. We obtain necessary and sufficient conditions under which such functions belong to the Laguerre-Pólya I class or, in our case, have only real negative zeros. In addition, we illustrate their relation to the partial theta function.
\end{abstract}

1. Introduction. To begin with, we provide the definitions of the Laguerre-Pólya class and Laguerre-Pólya class of type I.

Definition 1. A real entire function $f$ is said to be in the Laguerre-Pólya class, written $f \in \mathcal{L}$ - $\mathcal{P}$, if it can be expressed in the form

$$
f(z)=c z^{n} e^{-\alpha z^{2}+\beta z} \prod_{k=1}^{\infty}\left(1-\frac{z}{x_{k}}\right) e^{z x_{k}^{-1}},
$$

where $c, \alpha, \beta, x_{k} \in \mathbb{R}, x_{k} \neq 0, \alpha \geq 0, n$ is a nonnegative integer and $\sum_{k=1}^{\infty} x_{k}^{-2}<\infty$.

Definition 2. A real entire function $f$ is said to be in the Laguerre-Pólya class of type I, written $f \in \mathcal{L}-\mathcal{P} I$, if it can be expressed in the following form

$$
f(z)=c z^{n} e^{\beta z} \prod_{k=1}^{\infty}\left(1+\frac{z}{x_{k}}\right),
$$

where $c \in \mathbb{R}, \beta \geq 0, x_{k}>0, n$ is a nonnegative integer, and $\sum_{k=1}^{\infty} x_{k}^{-1}<\infty$.

Note that the product on the right-hand sides in both definitions can be finite or empty (in the latter case, the product equals 1 ).

These classes are essential in the theory of entire functions since it appears that the polynomials with only real zeros (or only real and nonpositive zeros) converge locally uniformly to these and only these functions. The following prominent theorem provides an even stronger result.

2010 Mathematics Subject Classification: 30C15; 30D15; 30D35; $26 \mathrm{C} 10$.

Keywords: Laguerre-Pólya class; entire functions of order zero; real-rooted polynomials; multiplier sequences; complex zero decreasing sequences.

doi:10.30970/ms.56.2.149-161

(C) T. H. Nguyen, A. Vishnyakova, 2021 
Theorem A (E. Laguerre and G. Pólya, see, for example, [5, p.42-46], [19, Ch.VIII, §3]). (i) Let $\left(P_{n}\right)_{n=1}^{\infty}, P_{n}(0)=1$, be a sequence of real polynomials having only real zeros which converges uniformly on the disc $|z| \leq A, A>0$. Then this sequence converges locally uniformly in $\mathbb{C}$ to an entire function from the $\mathcal{L}$ - $\mathcal{P}$ class.

(ii) For any $f \in \mathcal{L}$ - $\mathcal{P}$ there exists a sequence of real polynomials with only real zeros, which converges locally uniformly to $f$.

(iii) Let $\left(P_{n}\right)_{n=1}^{\infty}, P_{n}(0)=1$, be a sequence of real polynomials having only real negative zeros which converges uniformly on the disc $|z| \leq A, A>0$. Then this sequence converges locally uniformly in $\mathbb{C}$ to an entire function from the class $\mathcal{L}-\mathcal{P} I$.

(iv) For any $f \in \mathcal{L}-\mathcal{P} I$ there is a sequence of real polynomials with only real nonpositive zeros, which converges locally uniformly to $f$.

Further, we define the second quotients of Taylor coefficients of $f$.

Let $f(z)=\sum_{k=0}^{\infty} a_{k} z^{k}$ be an entire function with real nonzero coefficients, then

$$
q_{n}=q_{n}(f):=\frac{a_{n-1}^{2}}{a_{n-2} a_{n}}, \quad n \geq 2 .
$$

From this definition it follows straightforwardly that

$$
a_{n}=a_{1}\left(\frac{a_{1}}{a_{0}}\right)^{n-1} \frac{1}{q_{2}^{n-1} q_{3}^{n-2} \cdot \ldots \cdot q_{n-1}^{2} q_{n}}, \quad n \geq 2 .
$$

In general, the problem of understanding whether a given entire function has only real zeros is not trivial. However, in 1926, J. I. Hutchinson found the following simple sufficient condition in terms of coefficients for an entire function with positive coefficients to have only real zeros.

Theorem B (J. I. Hutchinson [6]). Let $f(x)=\sum_{k=0}^{\infty} a_{k} x^{k}, a_{k}>0$ for all $k$. Then $q_{n}(f) \geq 4$, for all $n \geq 2$, if and only if the following two conditions are fulfilled:

$(i)$ The zeros of $f(x)$ are all real, simple and negative,

ii) the zeros of any polynomial $\sum_{k=m}^{n} a_{k} x^{k}, m<n$, formed by taking any number of consecutive terms of $f(x)$, are all real and non-positive.

For some extensions of Hutchinson's results see, for example, [3, §4].

Next, we define the multiplier sequence.

Definition 3. A sequence $\left(\gamma_{k}\right)_{k=0}^{\infty}$ of real numbers is called a multiplier sequence if, whenever the real polynomial $P(x)=\sum_{k=0}^{n} a_{k} z^{k}$ has only real zeros, the polynomial $\sum_{k=0}^{n} \gamma_{k} a_{k} z^{k}$ has only real zeros. The class of multiplier sequences is denoted by $\mathcal{M S}$.

The following theorem fully describes multiplier sequences.

Theorem C (G. Pólya and J.Schur, cf. [28], [27, p.100-124], [26, p.29-47]). Let $\left(\gamma_{k}\right)_{k=0}^{\infty}$ be a given real sequence. The following three statements are equivalent:

1. $\left(\gamma_{k}\right)_{k=0}^{\infty}$ is a multiplier sequence.

2. For every $n \in \mathbb{N}$ the polynomial $P_{n}(z)=\sum_{k=0}^{n}\left(\begin{array}{l}n \\ k\end{array}\right) \gamma_{k} z^{k}$ has only real zeros of the same sign.

3. The power series $\Phi(z):=\sum_{k=0}^{\infty} \frac{\gamma_{k}}{k !} z^{k}$ converges absolutely in the whole complex plane and the entire function $\Phi(z)$ or the entire function $\Phi(-z)$ admits the representation

$$
C e^{\sigma z} z^{m} \prod_{k=1}^{\infty}\left(1+\frac{z}{x_{k}}\right),
$$

where $C \in \mathbb{R}, \sigma \geq 0, m \in \mathbb{N} \cup\{0\}, 0<x_{k} \leq \infty$, and $\sum_{k=1}^{\infty} 1 / x_{k}<\infty$.

Strikingly, the following fact is an obvious consequence. 
Corollary of Theorem C. The sequence $\left(\gamma_{0}, \gamma_{1}, \ldots, \gamma_{l}, 0,0, \ldots\right)$ is a multiplier sequence if and only if the polynomial $P(z)=\sum_{k=0}^{l} \frac{\gamma_{k}}{k !} z^{k}$ has only real zeros of the same sign.

Further, let us introduce the notion of a complex zero decreasing sequence. For a real polynomial $P$ we denote by $Z_{\mathbb{C}}(P)$ the number of nonreal zeros of $P$ counting multiplicities.

Definition 4. A sequence $\left(\gamma_{k}\right)_{k=0}^{\infty}$ of real numbers is said to be a complex zero decreasing sequence (we write $\left(\gamma_{k}\right)_{k=0}^{\infty} \in \mathcal{C} \mathcal{Z D S}$ ), if

$$
Z_{\mathbb{C}}\left(\sum_{k=0}^{n} \gamma_{k} a_{k} z^{k}\right) \leq Z_{\mathbb{C}}\left(\sum_{k=0}^{n} a_{k} z^{k}\right),
$$

for any real polynomial $\sum_{k=0}^{n} a_{k} z^{k}$.

The existence of nontrivial $\mathcal{C Z D S}$ sequences is a consequence of the following remarkable theorem proved by Laguerre and extended by Pólya.

Theorem D (E. Laguerre, see [27, p.314-321]). Let $f$ be an entire function from the LaguerrePólya class having only negative zeros. Then $(f(k))_{k=0}^{\infty} \in \mathcal{C Z D S}$.

As it follows from the theorem above,

$$
\left(a^{-k^{2}}\right)_{k=0}^{\infty} \in \mathcal{C} \mathcal{Z} \mathcal{D} \mathcal{S}, a \geq 1, \quad\left(\frac{1}{k !}\right)_{k=0}^{\infty} \in \mathcal{C} \mathcal{Z} \mathcal{D} \mathcal{S}
$$

A special entire function $g_{a}(z)=\sum_{k=0}^{\infty} z^{k} a^{-k^{2}}, a>1$, known as the partial theta function (the classical Jacobi theta function is defined by the series $\theta(z):=\sum_{k=-\infty}^{\infty} z^{k} a^{-k^{2}}$ ), was investigated by many mathematicians. Note that $q_{n}\left(g_{a}\right)=a^{2}$ for all $n$. The survey [30] by S.O. Warnaar contains the history of investigation of the partial theta-function and some of its main properties.

Note that, since $\left(a^{-k^{2}}\right)_{k=0}^{\infty} \in \mathcal{C Z D S}$ for $a \geq 1$, we conclude that for every $n \geq 2$ there exists a constant $c_{n}>1$ such that $S_{n}\left(z, g_{a}\right):=\sum_{k=0}^{n} z^{k} a^{-k^{2}} \in \mathcal{L}-\mathcal{P} \Leftrightarrow a^{2} \geq c_{n}$. The following theorem answers the question for which values of a parameter $a$ the partial theta-function and its Taylor sections belong to the $\mathcal{L}-\mathcal{P}$ class.

Theorem E (O. Katkova, T. Lobova, A. Vishnyakova [7]). There exists a constant $q_{\infty}$ $\left(q_{\infty} \approx 3.23363666 \ldots\right)$ such that:

(1) $g_{a}(z) \in \mathcal{L}-\mathcal{P} \Leftrightarrow a^{2} \geq q_{\infty}$;

(2) $g_{a}(z) \in \mathcal{L}-\mathcal{P} \Leftrightarrow$ there exists $z_{0} \in\left(-a^{3},-a\right)$ such that $g_{a}\left(z_{0}\right) \leq 0$;

(3) for a given $n \geq 2$, we have $S_{n}\left(z, g_{a}\right) \in \mathcal{L}-\mathcal{P} \Leftrightarrow$ there exists $z_{n} \in\left(-a^{3},-a\right)$ such that $S_{n}\left(z_{n}, g_{a}\right) \leq 0$

(4) $4=c_{2}>c_{4}>c_{6}>\cdots$ and $\lim _{n \rightarrow \infty} c_{2 n}=q_{\infty}$;

(5) $3=c_{3}<c_{5}<c_{7}<\cdots$ and $\lim _{n \rightarrow \infty} c_{2 n+1}=q_{\infty}$.

We would like to mention a series of works by V. P. Kostov dedicated to the interesting properties of zeros of the partial theta-function and its derivative (see [9-17]). Besides, a wonderful paper [18] among the other results explains the role of the constant $q_{\infty}$ in the study of the set of entire functions with positive coefficients having all Taylor truncations with only real zeros. See also [29] for the properties of the leading root of the partial theta function.

Subsequently, we need the following Lemma from the work [21].

Lemma F (see [21, Lemma 2.1] or [22, Lemma 1.2]). If $f(z)=\sum_{k=0}^{\infty} a_{k} z^{k}, a_{k}>0$, belongs to $\mathcal{L}-\mathcal{P} I$, then $q_{3}\left(q_{2}-4\right)+3 \geq 0$. In particular, if $q_{3} \geq q_{2}$, then $q_{2} \geq 3$. 
It appears that for many important entire functions with positive coefficients $f(z)=$ $\sum_{k=0}^{\infty} a_{k} z^{k}$ (for example, the partial theta function from [7], functions from [2] and [1], the $q$-Kummer function ${ }_{1} \phi_{1}(q ;-q ; q,-z)$ and others) the following two conditions are equivalent: (i) $f \in \mathcal{L}-\mathcal{P} I ;(i i)$ there exists $x_{0} \in\left[-\frac{a_{1}}{a_{2}}, 0\right]$ such that $f\left(x_{0}\right) \leq 0$.

The following theorem is a necessary condition for an entire function to belong to the Laguerre-Polýa class of type I, in terms of the closest to zero roots. We will further use it in our proofs.

Theorem G (T. H. Nguyen, A. Vishnyakova [22]). Let $f(z)=\sum_{k=0}^{\infty} a_{k} z^{k}, a_{k}>0$ for all $k$, be an entire function. Suppose that the quotients $q_{n}(f)$ satisfy the following condition: $q_{2}(f) \leq q_{3}(f)$. If $f \in \mathcal{L}-\mathcal{P} I$, i.e. belongs to the Laguerre-Pólya class of type $I$, then there exists $z_{0} \in\left[-\frac{a_{1}}{a_{2}}, 0\right]$ such that $f\left(z_{0}\right) \leq 0$.

Many important entire functions with monotonic second quotients of Taylor coefficients were previously studied. For instance, $f(z)=\sum_{k=0}^{\infty} \frac{z^{k}}{(k !) a^{k^{2}}}, a \geq 1$, has $q_{k}(f)=\frac{k}{k-1} a^{2}$ which are decreasing in $k$. It is known that $f \in \mathcal{L}-\mathcal{P} \mathcal{I}$ for all $a \geq 1$. In [8], it is proved that all Taylor sections of this function belong to the Laguerre-Pólya class of type I if and only if $a^{2} \geq q_{\infty}$. The following function is known as the second $q$-exponential function $E_{q}(z)$ with $q=1 / a$ :

$$
h_{a}(z)=1+\sum_{k=1}^{\infty} \frac{z^{k}}{\left(a^{k}-1\right)\left(a^{k-1}-1\right) \cdot \ldots \cdot(a-1)}=\prod_{k=1}^{\infty}\left(1+\frac{z}{a^{k}}\right), a>1 .
$$

We have $q_{k}\left(h_{a}\right)=\frac{a^{k}-1}{a^{k-1}-1}$, and $q_{k}\left(h_{a}\right)$ are decreasing in $k$. Note that this function has only real negative zeros, i.e. it belongs to the Laguerre-Pólya I class. Next, the function $\varphi_{m, a}(z)=$ $\sum_{k=0}^{\infty} \frac{z^{k}}{a^{k^{2}}}(k !)^{m}, a>1, m \geq 1$ was studied by A. Bohdanov and A. Vishnyakova in [2]. Note that $q_{k}\left(\varphi_{m, a}\right)$ are increasing in $k$. The necessary and sufficient conditions for this function to belong to the Laguerre-Pólya class of type I were investigated. The function

$$
y_{a}(z)=\sum_{k=0}^{\infty} \frac{z^{k}}{(a+1)\left(a^{2}+1\right) \cdot \ldots \cdot\left(a^{k}+1\right)}, a>1,
$$

is also known as the $q$-Kummer function ${ }_{1} \phi_{1}(q ;-q ; q,-z)$, where $q=1 / a$ (see [4], formula $(1.2 .22)$ ). Note that its $q_{k}$ are increasing in $k$. The question of its belonging to the LaguerrePólya class of type I was investigated by T.H. Nguyen in [25].

In [20], we have proved that if an entire function with positive coefficients has a decreasing sequence of second quotients of Taylor coefficients and the limit of this sequence is greater than or equal to $q_{\infty}$, then this function belongs to the Laguerre-Pólya class of type I. In [21], [23] and [24], we have found the conditions for entire functions with increasing second quotients of Taylor coefficients to belong to the Laguerre-Pólya class of type I.

In this paper, we study the entire functions with non-monotonic second quotients and find the conditions for them to belong to the Laguerre-Pólya class of type I. We present our first attempt at investigating entire functions with non-monotonic second quotients and consider the following function

$$
f(x)=\sum_{k=0}^{\infty} \frac{x^{k}}{q_{2}^{k-1} q_{3}^{k-2} \cdot \ldots \cdot q_{k-1}^{2} q_{k}}
$$

where $q_{2}=q_{4}=q_{6}=\ldots=a>1, q_{3}=q_{5}=q_{7}=\ldots=b>1$, or

$$
f(x)=1+x+\frac{x^{2}}{a}+\frac{x^{3}}{a^{2} b}+\frac{x^{4}}{a^{3} b^{2} a}+\frac{x^{5}}{a^{4} b^{3} a^{2} b}+\ldots
$$

We look into the case when $a \neq b$. Note that when $a=b$, we obtain the case of the 
partial-theta function. We find the new conditions for which $(a, b)$ the entire function $f$ belongs to the Laguerre-Pólya I class.

By Lemma $\mathrm{F}$, if $f \in \mathcal{L}-\mathcal{P} I, q_{2}(f)=a, q_{3}(f)=b$ and $a<b$, then $a \geq 3$.

Therefore, we look for $(a, b)$ such that $a<b$ and $a \geq 3$. The case $4 \leq a<b$ was studied by Hutchinson (see Theorem B), so we find new conditions for $a \in[3,4)$.

We present our main result.

Theorem 1. Let $f(z)=\sum_{k=0}^{\infty} a_{k} z^{k}, a_{k}>0$ for all $k$, be an entire function. Suppose that the quotients $q_{n}(f)$ satisfy the following condition: $q_{2}(f)=q_{4}(f)=q_{6}(f)=\ldots=a$, and $q_{3}(f)=q_{5}(f)=q_{7}(f)=\ldots=b, 1<a<b$. Then the function $f \in \mathcal{L}-\mathcal{P} I$ if and only if there exists $z_{0} \in\left[-\frac{a_{1}}{a_{2}}, 0\right]$ such that $f\left(z_{0}\right) \leq 0$.

The following theorem contains a sufficient condition for the existence of such a point $z_{0}$ for the case $q_{2}(f)<4$.

Theorem H (T. H. Nguyen, A. Vishnyakova [22]). Let $f(z)=\sum_{k=0}^{\infty} a_{k} z^{k}, a_{k}>0$ for all $k$, be an entire function and $3 \leq q_{2}(f)<4, q_{3}(f) \geq 2$, and $q_{4}(f) \geq 3$. If $q_{3}(f) \leq \frac{8}{d(4-d)}$, where $d=\min \left(q_{2}(f), q_{4}(f)\right)$, then there exists $z_{0} \in\left[-\frac{a_{1}}{a_{2}}, 0\right]$ such that $f\left(z_{0}\right) \leq 0$.

Hence, in our case, the sufficient condition is $b \leq \frac{8}{a(4-a)}$.

The following theorem gives a necessary condition for an entire function to belong to the Laguerre-Polýa class of type I, in terms of the second quotients of its Taylor coefficients $q_{n}$. Theorem I (T. H. Nguyen, A. Vishnyakova [22]). Let $f(z)=\sum_{k=0}^{\infty} a_{k} z^{k}, a_{k}>0$ for all $k$. If $f \in \mathcal{L}-\mathcal{P} I, q_{2}(f)<4$ and $q_{2}(f) \leq q_{3}(f)$, then

$$
q_{3}(f) \leq \frac{-q_{2}(f)\left(2 q_{2}(f)-9\right)+2\left(q_{2}(f)-3\right) \sqrt{q_{2}(f)\left(q_{2}(f)-3\right)}}{q_{2}(f)\left(4-q_{2}(f)\right)} .
$$

Thus, in our case, the necessary condition is

$$
b \leq \frac{-a(2 a-9)+2(a-3) \sqrt{a(a-3)}}{a(4-a)} .
$$

Our next result is the Theorem 2 below.

Theorem 2. Let $f_{a, b}(z)=\sum_{k=0}^{\infty} a_{k} z^{k}, a_{k}>0$ for all $k$, be an entire function such that $q_{2}(f)=q_{4}(f)=q_{6}(f)=\ldots=a$, and $q_{3}(f)=q_{5}(f)=q_{7}(f)=\ldots=b, 1<a<b$. Then the following statements are valid:

1. If $f \in \mathcal{L}-\mathcal{P} I$, then $a \geq q_{\infty}$.

2. If the numbers $a, b, q_{\infty} \leq a<b$, are such that $f_{a, b} \in \mathcal{L}-\mathcal{P} I$, then for every $c, a<c<b$ we have $f_{a, c} \in \mathcal{L}-\mathcal{P} I$.

3. If the numbers $a, b, q_{\infty} \leq a<b$, are such that $f_{a, b} \in \mathcal{L}-\mathcal{P}$, then for every $d, a<d<b$ we have $f_{d, b} \in \mathcal{L}-\mathcal{P} I$.

Note that, by Theorem $\mathrm{C}$ and Theorem $\mathrm{D}$, every new entire function from the $\mathcal{L}-\mathcal{P} I$ class generates a new multiplier sequence and a new complex zero decreasing sequence. So, we obtain the following direct corollary from Theorem 1 .

Corollary 1. Let $f(z)=\sum_{k=0}^{\infty} a_{k} z^{k}, a_{k}>0$ for all $k$, be an entire function. Suppose that the quotients $q_{n}(f)$ satisfy the following condition: $q_{2}(f)=q_{4}(f)=q_{6}(f)=\ldots=a$, and $q_{3}(f)=q_{5}(f)=q_{7}(f)=\ldots=b, 1<a<b$. Suppose that there exists $z_{0} \in\left[-\frac{a_{1}}{a_{2}}, 0\right]$ such that $f\left(z_{0}\right) \leq 0$ (for example, this condition is fulfilled if $\left.a<b \leq \frac{8}{a(4-a)}\right)$. Then $\left(k ! a_{k}\right)_{k=0}^{\infty} \in \mathcal{M S}$ and $(f(k))_{k=0}^{\infty} \in \mathcal{C} \mathcal{Z} \mathcal{D} \mathcal{S}$. 


\section{Proof of Theorem 1.}

Proof. For an entire function $f(z)=\sum_{k=0}^{\infty} a_{k} z^{k}$ with real positive coefficients, without loss of generality, we can assume that $a_{0}=a_{1}=1$, since we can consider a function $g(x)=$ $a_{0}^{-1} f\left(a_{0} a_{1}^{-1} x\right)$ instead of $f(x)$, due to the fact that such rescaling of $f$ preserves its property of having real zeros and preserves the second quotients: $q_{n}(g)=q_{n}(f)$ for all $n$. Throughout the paper, we use notation $p_{n}$ and $q_{n}$ instead of $p_{n}(f)$ and $q_{n}(f)$.

Thereafter, we consider a function

$$
\varphi(x)=f(-x)=1-x+\sum_{k=2}^{\infty} \frac{(-1)^{k} x^{k}}{q_{2}^{k-1} q_{3}^{k-2} \cdot \ldots \cdot q_{k-1}^{2} q_{k}}
$$

instead of $f$.

Firstly, in our case, $q_{2}=a, q_{3}=b, a<b$, according to Theorem $\mathrm{F}$, if $\varphi \in \mathcal{L}-\mathcal{P} I$, then there exists such a point $z_{0} \in\left[0,-\frac{a_{1}}{a_{2}}\right]=[0, a]$ that $\varphi\left(z_{0}\right) \leq 0$.

To prove the converse statement, we need the following lemma.

Lemma 1. Let $\varphi(x)=\sum_{k=0}^{\infty}(-1)^{k} a_{k} x^{k}, a_{k}>0, k=0,1,2, \ldots$, be an entire function such that $q_{2}=q_{4}=q_{6}=\ldots=a \geq 3, q_{3}=q_{5}=q_{7}=\ldots=b>a$. For an arbitrary integer $j \geq 2$, we define $\rho_{j}:=q_{2} q_{3} \cdot \ldots \cdot q_{j} \sqrt{q_{j+1}}$. Then, for all even $j=2 s, s \in \mathbb{N}$, the function $\varphi$ has exactly $j$ zeros on the disk $\left\{x:|x|<\rho_{j}\right\}$ counting multiplicities.

Proof of Lemma 1. For $j \geq 2$, we have

$$
\varphi(x)=\sum_{k=0}^{\infty} \frac{(-1)^{k} x^{k}}{q_{2}^{k-1} q_{3}^{k-2} \cdot \ldots \cdot q_{k}}=\left(\sum_{k=0}^{j-3}+\sum_{k=j-2}^{j+2}+\sum_{k=j+3}^{\infty}\right)=\Sigma_{1, j}(x)+g_{j}(x)+\Sigma_{2, j}(x) .
$$

In more details,

$$
\begin{gathered}
g_{j}(x)=\frac{(-1)^{j-2} x^{j-2}}{q_{2}^{j-3} q_{3}^{j-4} \cdot \ldots \cdot q_{j-2}}+\frac{(-1)^{j-1} x^{j-1}}{q_{2}^{j-2} q_{3}^{j-3} \cdot \ldots \cdot q_{j-2}^{2} q_{j-1}}+\frac{(-1)^{j} x^{j}}{q_{2}^{j-1} q_{3}^{j-2} \cdot \ldots \cdot q_{j-2}^{3} q_{j-1}^{2} q_{j}}+ \\
+\frac{(-1)^{j+1} x^{j+1}}{q_{2}^{j} q_{3}^{j-1} \cdot \ldots \cdot q_{j-2}^{4} q_{j-1}^{3} q_{j}^{2} q_{j+1}}+\frac{(-1)^{j+2} x^{j+2}}{q_{2}^{j+1} q_{3}^{j} \cdot \ldots \cdot q_{j-2}^{5} q_{j-1}^{4} q_{j}^{3} q_{j+1}^{2} q_{j+2}}= \\
=\frac{(-1)^{j-2} x^{j-2}}{q_{2}^{j-3} q_{3}^{j-4} \cdot \ldots \cdot q_{j-2}} \cdot\left(1-\frac{x}{q_{2} q_{3} \cdot \ldots \cdot q_{j-2} q_{j-1}}+\right. \\
\left.+\frac{x^{2}}{q_{2}^{2} q_{3}^{2} \cdot \ldots \cdot q_{j-2}^{2} q_{j-1}^{2} q_{j}}-\frac{x^{4}}{q_{2}^{3} q_{3}^{3} \cdot \ldots \cdot q_{j-2}^{3} q_{j-1}^{3} q_{j}^{2} q_{j+1}}+\frac{x_{2}^{4}}{q_{2}^{4} q_{3}^{4} \cdot \ldots \cdot q_{j-2}^{4} q_{j-1}^{4} q_{j}^{3} q_{j+1}^{2} q_{j+2}}\right) .
\end{gathered}
$$

By the definition of $\rho_{j}$, we have $q_{2} q_{3} \cdot \ldots \cdot q_{j}<\rho_{j}<q_{2} q_{3} \cdot \ldots \cdot q_{j} q_{j+1}$. We get

$$
\begin{gathered}
g_{j}\left(\rho_{j} e^{i \theta}\right)=(-1)^{j-2} e^{i(j-2) \theta} q_{2} q_{3}^{2} \cdot \ldots \cdot q_{j-2}^{j-3} q_{j-1}^{j-2} q_{j}^{j-2} q_{j+1}^{\frac{j-2}{2}} \cdot\left(1-q_{j} \sqrt{q_{j+1}} e^{i \theta}+q_{j} q_{j+1} e^{2 i \theta}-\right. \\
\left.-q_{j} \sqrt{q_{j+1}} e^{3 i \theta}+\frac{q_{j}}{q_{j+2}} e^{4 i \theta}\right)=(-1)^{j-2} e^{i(j-2) \theta} q_{2} q_{3}^{2} \cdot \ldots \cdot q_{j-2}^{j-3} q_{j-1}^{j-2} q_{j}^{j-2} q_{j+1}^{\frac{j-2}{2}} \times \\
\times\left(1-e^{i \theta} q_{j} \sqrt{q_{j+1}}+e^{2 i \theta} q_{j} q_{j+1}-e^{3 i \theta} q_{j} \sqrt{q_{j+1}}+e^{4 i \theta}\right),
\end{gathered}
$$

since, by our assumptions, $q_{j}=q_{j+2}$.

Our aim is to show that for every even $j$ the following inequality holds:

$$
\min _{0 \leq \theta \leq 2 \pi}\left|g_{j}\left(\rho_{j} e^{i \theta}\right)\right|>\max _{0 \leq \theta \leq 2 \pi}\left|\varphi\left(\rho_{j} e^{i \theta}\right)-g_{j}\left(\rho_{j} e^{i \theta}\right)\right|,
$$


so that the number of zeros of $\varphi$ in the circle $\left\{x:|x|<\rho_{j}\right\}$ is equal to the number of zeros of $g_{j}$ in the same circle. Subsequently in the proof, we also find the number of zeros of $g_{j}$ in this circle. First, we find $\min _{0 \leq \theta \leq 2 \pi}\left|g_{j}\left(\rho_{j} e^{i \theta}\right)\right|$. We obtain

$$
\begin{gathered}
g_{j}\left(\rho_{j} e^{i \theta}\right)=(-1)^{j-2} e^{i j \theta} q_{2} q_{3}^{2} \cdot \ldots \cdot q_{j-2}^{j-3} q_{j-1}^{j-2} q_{j}^{j-2} q_{j+1}^{\frac{j-2}{2}}\left(2 \cos 2 \theta-2 \cos \theta q_{j} \sqrt{q_{j+1}}+q_{j} q_{j+1}\right)= \\
=:(-1)^{j-2} e^{i j \theta} q_{2} q_{3}^{2} \cdot \ldots \cdot q_{j-2}^{j-3} q_{j-1}^{j-2} q_{j}^{j-2} q_{j+1}^{\frac{j-2}{2}} \cdot \psi_{j}(\theta) .
\end{gathered}
$$

We consider $\psi_{j}(\theta)$ as following: $\psi_{j}(\theta)=\widetilde{\psi}_{j}(t):=4 t^{2}-2 q_{j} \sqrt{q_{j+1}} t+\left(q_{j} q_{j+1}-2\right)$, where $t:=\cos \theta$, and where we have used that $\cos 2 \theta=2 t^{2}-1$.

The vertex of the parabola is $t_{j}=q_{j} \sqrt{q_{j+1}} / 4$. Under our assumptions, $q_{2}=q_{4}=q_{6}=$ $\ldots=a \geq 3$, and $q_{3}=q_{5}=q_{7}=\ldots=b>a$, so that $t_{j}>1$. Hence, $\min _{t \in[-1,1]} \widetilde{\psi}_{j}(t)=$ $\widetilde{\psi}_{j}(1)=2-2 q_{j} \sqrt{q_{j+1}}+q_{j} q_{j+1}=q_{j} \sqrt{q_{j+1}}\left(\sqrt{q_{j+1}}-2\right)+2$. We want to show that $\widetilde{\psi}_{j}(1)>0$, whence $\min _{t \in[-1,1]}\left|\widetilde{\psi}_{j}(t)\right|=\widetilde{\psi}_{j}(1)$. If $q_{j+1} \geq 4$, then $q_{j} \sqrt{q_{j+1}}\left(\sqrt{q_{j+1}}-2\right)+2>0$. If $q_{j+1}<4$, taking into account the fact that $j$ is an even integer, we have $q_{j}=a, q_{j+1}=b, a<b<4$, and then

$$
q_{j} \sqrt{q_{j+1}}\left(\sqrt{q_{j+1}}-2\right)+2=a \sqrt{b}(\sqrt{b}-2)+2 \geq a \sqrt{a}(\sqrt{a}-2)+2=a^{2}-2 a \sqrt{a}+2 .
$$

Next, denote by $y=\sqrt{a} \geq 0$, and $g(y)=y^{4}-2 y^{3}+2$. It is easy to calculate that $\min _{y \geq 0} g(y)=g\left(\frac{3}{2}\right)=\frac{5}{16}>0$. Consequently, we get $2-2 q_{j} \sqrt{q_{j+1}}+q_{j} q_{j+1}>0$. Thus, $\widetilde{\psi}_{j}(t)>0$ for all $t \in[-1,1]$, and we have obtained the estimate from below:

$$
\min _{0 \leq \theta \leq 2 \pi}\left|g_{j}\left(\rho_{j} e^{i \theta}\right)\right|=q_{2} q_{3}^{2} \cdot \ldots \cdot q_{j-2}^{j-3} q_{j-1}^{j-2} q_{j}^{j-2} q_{j+1}^{\frac{j-2}{2}}\left(2-2 q_{j} \sqrt{q_{j+1}}+q_{j} q_{j+1}\right) .
$$

Second, we estimate the modulus of $\Sigma_{1}$ from above. We have

$$
\left|\Sigma_{1}\left(\rho_{j} e^{i \theta}\right)\right| \leq \sum_{k=0}^{j-3} \frac{q_{2}^{k} q_{3}^{k} \cdot \ldots \cdot q_{j}^{k} q_{j+1}^{\frac{k}{2}}}{q_{2}^{k-1} q_{3}^{k-2} \cdot \ldots \cdot q_{k}} .
$$

We rewrite the sum from right and estimate the finite sum from above by the sum of the infinite geometric progression

$$
\begin{gathered}
\left|\Sigma_{1}\left(\rho_{j} e^{i \theta}\right)\right| \leq\left(q_{2} q_{3}^{2} \cdot \ldots \cdot q_{j-3}^{j-4} q_{j-2}^{j-3} q_{j-1}^{j-3} q_{j}^{j-3} q_{j+1}^{\frac{j-3}{2}}+q_{2} q_{3}^{2} \cdot \ldots \cdot q_{j-4}^{j-5} q_{j-3}^{j-4} q_{j-2}^{j-4} q_{j-1}^{j-4} q_{j}^{j-4} q_{j+1}^{\frac{j-4}{2}}+\right. \\
\left.\quad+q_{2} q_{3}^{2} \cdot \ldots \cdot q_{j-5}^{j-6} q_{j-4}^{j-5} q_{j-3}^{j-5} q_{j-2}^{j-5} q_{j-1}^{j-5} q_{j}^{j-5} q_{j+1}^{\frac{j-5}{2}}+\cdots\right)= \\
=q_{2} q_{3}^{2} \cdot \ldots \cdot q_{j-3}^{j-4} q_{j-2}^{j-3} q_{j-1}^{j-3} q_{j}^{j-3} q_{j+1}^{\frac{j-3}{2}}\left(1+\frac{1}{q_{j-2} q_{j-1} q_{j} \sqrt{q_{j+1}}}+\frac{1}{q_{j-3} q_{j-2}^{2} q_{j-1}^{2} q_{j}^{2}\left(\sqrt{q_{j+1}}\right)^{2}}+\cdots\right) \\
\leq q_{2} q_{3}^{2} \cdot \ldots \cdot q_{j-3}^{j-4} q_{j-2}^{j-3} q_{j-1}^{j-3} q_{j}^{j-3} q_{j+1}^{\frac{j-3}{2}} \cdot \frac{1}{1-\frac{1}{q_{j-2} q_{j-1} q_{j} \sqrt{q_{j+1}}}} .
\end{gathered}
$$

Finally, we obtain

$$
\left|\Sigma_{1}\left(\rho_{j} e^{i \theta}\right)\right| \leq q_{2} q_{3}^{2} \cdot \ldots \cdot q_{j-3}^{j-4} q_{j-2}^{j-3} q_{j-1}^{j-3} q_{j}^{j-3} q_{j+1}^{\frac{j-3}{2}} \cdot \frac{1}{1-\frac{1}{q_{j-2} q_{j-1} q_{j} \sqrt{q_{j+1}}}} .
$$

Next, the estimation of $\left|\Sigma_{2}\left(\rho_{j} e^{i \theta}\right)\right|$ from above can be made analogously:

$$
\begin{aligned}
& \left|\Sigma_{2}\left(\rho_{j} e^{i \theta}\right)\right| \leq \sum_{k=j+3}^{\infty} \frac{q_{2}^{k} q_{3}^{k} \cdot \ldots \cdot q_{j}^{k} q_{j+1}^{\frac{k}{2}}}{q_{2}^{k-1} q_{3}^{k-2} \cdot \ldots \cdot q_{k}}=\frac{q_{2} q_{3}^{2} \cdot \ldots \cdot q_{j}^{j-1} q_{j+1}^{\frac{j-3}{2}}}{q_{j+2}^{2} q_{j+3}} \times \\
& \times\left(1+\frac{1}{\sqrt{q_{j+1}} q_{j+2} q_{j+3} q_{j+4}}+\frac{1}{\left(\sqrt{q_{j+1}}\right)^{2} q_{j+2}^{2} q_{j+3}^{2} q_{j+4}^{2} q_{j+5}}+\cdots\right) .
\end{aligned}
$$


The latter can be estimated from above by the sum of the geometric progression, so, we obtain

$$
\left|\Sigma_{2}\left(\rho_{j} e^{i \theta}\right)\right| \leq \frac{q_{2} q_{3}^{2} \cdot \ldots \cdot q_{j}^{j-1} q_{j+1}^{\frac{j-3}{2}}}{q_{j+2}^{2} q_{j+3}} \cdot \frac{1}{1-\frac{1}{\sqrt{q_{j+1}} q_{j+2} q_{j+3} q_{j+4}}} .
$$

Therefore, the desired inequality $\min _{0 \leq \theta \leq 2 \pi}\left|g_{j}\left(\rho_{j} e^{i \theta}\right)\right|>\max _{0 \leq \theta \leq 2 \pi}\left|\varphi\left(\rho_{j} e^{i \theta}\right)-g_{j}\left(\rho_{j} e^{i \theta}\right)\right|$ follows from

$$
\begin{gathered}
q_{2} q_{3}^{2} \cdot \ldots \cdot q_{j-2}^{j-3} q_{j-1}^{j-2} q_{j}^{j-2} q_{j+1}^{\frac{j-2}{2}} \cdot\left(2-2 q_{j} \sqrt{q_{j+1}}+q_{j} q_{j+1}\right)> \\
>q_{2} q_{3}^{2} \cdot \ldots \cdot q_{j-3}^{j-4} q_{j-2}^{j-3} q_{j-1}^{j-3} q_{j}^{j-3} q_{j+1}^{\frac{j-3}{2}} \cdot \frac{1}{1-\frac{1}{q_{j-2} q_{j-1} q_{j} \sqrt{q_{j+1}}}} \\
+\frac{q_{2} q_{3}^{2} \cdot \ldots \cdot q_{j}^{j-1} q_{j+1}^{\frac{j-3}{2}}}{q_{j+2}^{2} q_{j+3}} \cdot \frac{1}{1-\frac{1}{\sqrt{q_{j+1} q_{j+2} q_{j+3} q_{j+4}}}}
\end{gathered}
$$

Or, equivalently,

$$
\begin{gathered}
q_{j-1} q_{j} \sqrt{q_{j+1}}\left(2-2 q_{j} \sqrt{q_{j+1}}+q_{j} q_{j+1}\right)>\frac{1}{1-\frac{1}{q_{j-2} q_{j-1} q_{j} \sqrt{q_{j+1}}}}+ \\
+\frac{q_{j-1} q_{j}^{2}}{q_{j+2}^{2} q_{j+3}} \cdot \frac{1}{1-\frac{1}{\sqrt{q_{j+1}} q_{j+2} q_{j+3} q_{j+4}}}=\frac{1}{1-\frac{1}{q_{j-2} q_{j-1} q_{j} \sqrt{q_{j+1}}}}+\frac{1}{1-\frac{1}{\sqrt{q_{j+1}} q_{j+2} q_{j+3} q_{j+4}}}
\end{gathered}
$$

by our assumptions on the sequence $\left(q_{k}\right)_{k=2}^{\infty}$. Since $j$ is an even number, we have $q_{j-2}=q_{j}=$ $q_{j+2}=q_{j+4}=a$ and $q_{j-1}=q_{j+1}=q_{j+3}=b$, so the last inequality has the form

$$
b \sqrt{b} a(2-2 \sqrt{b} a+a b)>2\left(1-\frac{1}{b \sqrt{b} a^{2}}\right)^{-1} .
$$

Since $a \geq 3, b \geq 3$, the desired inequality (4) follows from $9 \sqrt{3}(2-2 \sqrt{b} a+a b)>$ $2\left(1-\frac{1}{27 \sqrt{3}}\right)^{-1}$ or, equivalently, $a b-2 \sqrt{b} a+2-\frac{6}{27 \sqrt{3}-1}>0$. After refactoring, we have $a(\sqrt{b}-1)^{2}-a+2-\frac{6}{27 \sqrt{3}-1}>0$. Next, we divide both sides of the inequality by $a$ and get $(\sqrt{b}-1)^{2}>1+\frac{8-54 \sqrt{3}}{a(27 \sqrt{3}-1)}$.

Since $8-54 \sqrt{3}<0$, the inequality above follows from $(\sqrt{a}-1)^{2}>1+\frac{8-54 \sqrt{3}}{4 \cdot(27 \sqrt{3}-1)}$.

Numerical calculations show that $1+\frac{8-54 \sqrt{3}}{4 \cdot(27 \sqrt{3}-1)}<0.53279$. Thus, the inequality is fulfilled for $\sqrt{a}>1.72993$, and, therefore, for $a>2.99266$. Under our assumptions, $a \geq 3$, so the inequality (4) is valid.

Consequently, we have proved that for all even $j$

$$
\min _{0 \leq \theta \leq 2 \pi}\left|g_{j}\left(\rho_{j} e^{i \theta}\right)\right|>\max _{0 \leq \theta \leq 2 \pi}\left|\varphi\left(\rho_{j} e^{i \theta}\right)-g_{j}\left(\rho_{j} e^{i \theta}\right)\right|,
$$

so the number of zeros of $\varphi$ in the circle $\left\{x:|x|<\rho_{j}\right\}$ is equal to the number of zeros of $g_{j}$ in this circle.

In the next stage of the proof, it remains to find the number of zeros of $g_{j}$ in the circle $\left\{x:|x|<\rho_{j}\right\}$.

Let us use the denotation $w=x \rho_{j}^{-1}$, so that $|w|<1$. This yields

$$
g_{j}\left(\rho_{j} w\right)=(-1)^{j-2} w^{j-2} q_{2} q_{3}^{2} \cdot \ldots \cdot q_{j-2}^{j-3} q_{j-1}^{j-2} q_{j}^{j-2} q_{j+1}^{\frac{j-2}{2}} \times
$$




$$
\times\left(1-q_{j} \sqrt{q_{j+1}} w+q_{j} q_{j+1} w^{2}-q_{j} \sqrt{q_{j+1}} w^{3}+w^{4}\right) .
$$

Therefore, it follows from (3) that $g_{j}$ does not have zeros on the circumference $\{x$ : $\left.|x|=\rho_{j}\right\}$, whence $g_{j}\left(\rho_{j} w\right)$ does not have zeros on the circumference $\{w:|w|=1\}$. Since $P_{j}(w)=1-q_{j} \sqrt{q_{j+1}} w+q_{j} q_{j+1} w^{2}-q_{j} \sqrt{q_{j+1}} w^{3}+w^{4}$ is a self-reciprocal polynomial in $w$, we can conclude that $P_{j}$ has exactly two zeros in the circle $\{w:|w|<1\}$. Hence, $g_{j}(x)$ has exactly $j$ zeros in the circle $\left\{x:|x|<\rho_{j}\right\}$ for all even $j$.

Lemma 2. Let $\varphi(x)=\sum_{k=0}^{\infty}(-1)^{k} a_{k} x^{k}, a_{k}>0, k=0,1,2, \ldots$, be an entire function such that $q_{2}=q_{4}=q_{6}=\ldots=a \geq 3, q_{3}=q_{5}=q_{7}=\ldots=b>a$. For an arbitrary integer $j \geq 2$ we define $\rho_{j}:=q_{2} q_{3} \cdot \ldots \cdot q_{j} \sqrt{q_{j+1}}$. Then for every even $j=2 s, s \in \mathbb{N}$, the following inequality holds: $\varphi\left(\rho_{j}\right) \geq 0$.

Proof. Since $\rho_{j} \in\left(q_{2} q_{3} \cdot \ldots \cdot q_{j}, q_{2} q_{3} \cdot \ldots \cdot q_{j} q_{j+1}\right)$, we have

$$
\begin{gathered}
1<\rho_{j}<\frac{\rho_{j}^{2}}{q_{2}}<\cdots<\frac{\rho_{j}^{j}}{q_{2}^{j-1} q_{3}^{j-2} \cdot \ldots \cdot q_{j}}, \\
\frac{\rho_{j}^{j}}{q_{2}^{j-1} q_{3}^{j-2} \cdot \ldots \cdot q_{j}}>\frac{\rho_{j}^{j+1}}{q_{2}^{j} q_{3}^{j-1} \cdot \ldots \cdot q_{j}^{2} q_{j+1}}>\frac{\rho_{j}^{j+2}}{q_{2}^{j+1} q_{3}^{j} \cdot \ldots \cdot q_{j}^{3} q_{j+1}^{2} q_{j+2}}>\cdots .
\end{gathered}
$$

Therefore, we get for even $j \geq 2$

$$
\varphi\left(\rho_{j}\right) \geq \sum_{k=j-3}^{j+3} \frac{(-1)^{k} \rho_{j}^{k}}{q_{2}^{k-1} q_{3}^{k-2} \cdot \ldots \cdot q_{k}}=: \mu_{j}\left(\rho_{j}\right),
$$

and it is sufficient to prove that for every even $j \geq 2$ we have $\mu_{j}\left(\rho_{j}\right) \geq 0$. After factoring out $\rho_{j}^{j-3} / q_{2}^{j-4} q_{3}^{j-5} \cdot \ldots \cdot q_{j-3}$ the desired inequality is expressed in the following form

$$
\begin{gathered}
-1+\frac{\rho_{j}}{q_{2} q_{3} \cdot \ldots \cdot q_{j-3} q_{j-2}}-\frac{\rho_{j}^{2}}{q_{2}^{2} q_{3}^{2} \cdot \ldots \cdot q_{j-2}^{2} q_{j-1}}+ \\
+\frac{\rho_{j}^{3}}{q_{2}^{3} q_{3}^{3} \cdot \ldots \cdot q_{j-2}^{3} q_{j-1}^{2} q_{j}}-\frac{\rho_{j}^{4}}{q_{2}^{4} q_{3}^{4} \cdot \ldots \cdot q_{j-2}^{4} q_{j-1}^{3} q_{j}^{2} q_{j+1}}+ \\
+\frac{\rho_{j}^{5}}{q_{2}^{5} q_{3}^{5} \cdot \ldots \cdot q_{j-2}^{5} q_{j-1}^{4} q_{j}^{3} q_{j+1}^{2} q_{j+2}}-\frac{\rho_{j}^{6}}{q_{2}^{6} q_{3}^{6} \cdot \ldots \cdot q_{k-2}^{6} q_{j-1}^{5} q_{j}^{4} q_{j+1}^{3} q_{j+2}^{2} q_{j+3}} \geq 0
\end{gathered}
$$

or, using that $\rho_{j}=q_{2} q_{3} \cdot \ldots \cdot q_{j} \sqrt{q_{j+1}}$,

$\nu_{j}\left(\rho_{j}\right):=-1+q_{j-1} q_{j} \sqrt{q_{j+1}}-2 q_{j-1} q_{j}^{2} q_{j+1}+q_{j-1} q_{j}^{2} q_{j+1} \sqrt{q_{j+1}}+\frac{q_{j-1} q_{j}^{2} \sqrt{q_{j+1}}}{q_{j+2}}-\frac{q_{j-1} q_{j}^{2}}{q_{j+2}^{2} q_{j+3}} \geq 0$.

By our assumptions on the sequence $\left(q_{k}\right)_{k=2}^{\infty}$, and, since $j$ is an even number, we have $q_{j}=q_{j+2}=a$ and $q_{j-1}=q_{j+1}=q_{j+3}=b$, so the last inequality has the form

$$
\nu_{j}\left(\rho_{j}\right):=-1+a b \sqrt{b}-2 a^{2} b^{2}+a^{2} b^{2} \sqrt{b}+a b \sqrt{b}-1=-2+2 a b \sqrt{b}-2 a^{2} b^{2}+a^{2} b^{2} \sqrt{b} \geq 0 .
$$

So we need the inequality $\nu_{j}\left(\rho_{j}\right)=a^{2} b^{2}(\sqrt{b}-2)+2(a b \sqrt{b}-1) \geq 0$.

Firstly, we consider the case when $b \geq 4$. We have $a^{2} b^{2}(\sqrt{b}-2) \geq 0$, and $(a b \sqrt{b}-1)>0$. Consequently, in the case $b \geq 4$, the desired inequality $\nu_{k}\left(\rho_{k}\right) \geq 0$ is proved.

Further, we consider the case $3 \leq a<b<4$. Under our assumptions we have $2<\frac{2}{9} a b$. Therefore, $\nu_{j}\left(\rho_{j}\right) \geq a^{2} b^{2} \sqrt{b}-2 a^{2} b^{2}+2 a b \sqrt{b}-\frac{2}{9} a b$. So, we want to check that $\psi(a, b):=a b \sqrt{b}-2 a b+2 \sqrt{b}-\frac{2}{9} \geq 0$. Since $3 \leq a<b<4$, we get 


$$
\psi(a, b)=a b(\sqrt{b}-2)+2 \sqrt{b}-\frac{2}{9} \geq b^{2}(\sqrt{b}-2)+2 \sqrt{b}-\frac{2}{9} .
$$

Set $\sqrt{b}=t, t \geq 0$, then we obtain the following inequality: $t^{5}-2 t^{4}+2 t-\frac{2}{9} \geq 0$.

This inequality holds for $t \geq 0.11126$ (we used numerical methods to find that the greatest real root of the polynomial on the left-hand side is less than 0.11126 ). Thus, it follows that it holds for $b \geq 0.01238$.

To prove Theorem 1, we need one more lemma.

Lemma 3. Let $\varphi(x)=\sum_{k=0}^{\infty}(-1)^{k} a_{k} x^{k}, a_{k}>0, k=0,1,2, \ldots$, be an entire function such that $q_{2}=q_{4}=q_{6}=\ldots=a \geq 3, q_{3}=q_{5}=q_{7}=\ldots=b>a$. Suppose that there exists $z_{0} \in\left[0, \frac{a_{1}}{a_{2}}\right]=[0, a]$ such that $\varphi\left(z_{0}\right) \leq 0$. For an arbitrary integer $j \geq 2$ we define $r_{j}:=q_{2} q_{3} \cdot \ldots \cdot q_{j} z_{0}$. Then the inequality $\varphi\left(r_{j}\right) \leq 0$ holds for every odd $j=2 m+1, m \in \mathbb{N}$.

Proof. Let us fix an arbitrary $j=2 m+1, m \in \mathbb{N}$. For $x \in[0,1]$ we have

whence $\varphi(x)>0$ for all $x \in[0,1]$.

$$
1 \geq x>\frac{x^{2}}{q_{2}}>\frac{x^{3}}{q_{2}^{2} q_{3}}>\frac{x^{4}}{q_{2}^{3} q_{3}^{2} q_{4}}>\cdots,
$$

Thus, we have $z_{0} \in(1, a]$, whence $q_{2} q_{3} \cdot \ldots \cdot q_{j}<r_{j} \leq q_{2} q_{3} \cdot \ldots \cdot q_{j} q_{j+1}$.

For all $x \in\left(q_{2} q_{3} \cdot \ldots \cdot q_{j}, q_{2} q_{3} \cdot \ldots \cdot q_{j} q_{j+1}\right]$, we have

$$
\begin{gathered}
1<x<\frac{x^{2}}{q_{2}}<\cdots<\frac{x^{j}}{q_{2}^{j-1} q_{3}^{j-2} \cdot \ldots \cdot q_{j}}, \\
\frac{x^{j}}{q_{2}^{j-1} q_{3}^{j-2} \cdot \ldots \cdot q_{j}} \geq \frac{x^{j+1}}{q_{2}^{j} q_{3}^{j-1} \cdot \ldots \cdot q_{j}^{2} q_{j+1}}>\frac{x^{j+2}}{q_{2}^{j+1} q_{3}^{j} \cdot \ldots \cdot q_{j}^{3} q_{j+1}^{2} q_{j+2}}>\cdots .
\end{gathered}
$$

Thus, for every $x \in\left(q_{2} q_{3} \cdot \ldots \cdot q_{j}, q_{2} q_{3} \cdot \ldots \cdot q_{j} q_{j+1}\right]$ we have

$$
\begin{gathered}
\varphi(x)=1-x+\frac{x^{2}}{q_{2}}-\ldots-\frac{x^{j-2}}{q_{2}^{j-3} q_{3}^{j-4} \cdot \ldots \cdot q_{j-2}}+\frac{x^{j-1}}{q_{2}^{j-2} q_{3}^{j-3} \cdot \ldots \cdot q_{j-2}^{2} q_{j-1}}- \\
-\frac{x^{j}}{q_{2}^{j-1} q_{3}^{j-2} \cdot \ldots \cdot q_{j-2}^{3} q_{j-1}^{2} q_{j}}+\frac{x^{j+1}}{q_{2}^{j} q_{3}^{j-1} \cdot \ldots \cdot q_{j-2}^{4} q_{j-1}^{3} q_{j}^{2} q_{j+1}}-\ldots \leq \frac{x^{j-1}}{q_{2}^{j-2} q_{3}^{j-3} \cdot \ldots \cdot q_{j-2}^{2} q_{j-1}}- \\
-\frac{x^{j+1}}{q_{2}^{j-1} q_{3}^{j-2} \cdot \ldots \cdot q_{j-2}^{3} q_{j-1}^{2} q_{j}}+\frac{x^{j-1}}{q_{2}^{j} q_{3}^{j-1} \cdot \ldots \cdot q_{j-2}^{4} q_{j-1}^{3} q_{j}^{2} q_{j+1}}-\ldots=\frac{x^{2}}{q_{2}^{j-2} q_{3}^{j-3} \cdot \ldots \cdot q_{j-2}^{2} q_{j-1}} \times \\
\times\left(1-\frac{x}{q_{2} q_{3} \cdot q_{j-1} q_{j}}+\frac{x^{3}}{q_{2}^{2} q_{3}^{2} \cdot q_{j-1}^{2} q_{j}^{2} q_{j+1}}-\frac{q_{2}^{3} q_{3}^{3} \cdot q_{j-1}^{3} q_{j}^{3} q_{j+1}^{2} q_{j+2}}{q^{2}}+\ldots\right) .
\end{gathered}
$$

Pasting $x=r_{j}=q_{2} q_{3} \cdot \ldots \cdot q_{j} z_{0}$, we obtain the inequality

$$
\begin{gathered}
\varphi\left(r_{j}\right) \leq \frac{r_{j}^{j-1}}{q_{2}^{j-2} q_{3}^{j-3} \cdot \ldots \cdot q_{j-2}^{2} q_{j-1}} \cdot\left(1-z_{0}+\frac{z_{0}^{2}}{q_{j+1}}-\frac{z_{0}^{3}}{q_{j+1}^{2} q_{j+2}}+\ldots\right)= \\
=\frac{r_{j}^{j-1}}{q_{2}^{j-2} q_{3}^{j-3} \cdot \ldots \cdot q_{j-2}^{2} q_{j-1}} \cdot\left(1-z_{0}+\frac{z_{0}^{2}}{a}-\frac{z_{0}^{3}}{a^{2} b}+\ldots\right)=\frac{r_{j}^{j-1}}{q_{2}^{j-2} q_{3}^{j-3} \cdot \ldots \cdot q_{j-2}^{2} q_{j-1}} \cdot \varphi\left(z_{0}\right) \leq 0 .
\end{gathered}
$$

Now, we are in a position to prove Theorem 1. Suppose that $\varphi(x)=\sum_{k=0}^{\infty}(-1)^{k} a_{k} x^{k}$, $a_{k}>0, k=0,1,2, \ldots, q_{2}=q_{4}=q_{6}=\ldots=a \geq 3, q_{3}=q_{5}=q_{7}=\ldots=b>a$, and there exists $z_{0} \in\left(1, \frac{a_{1}}{a_{2}}\right]=(1, a]$ such that $\varphi\left(z_{0}\right) \leq 0$.

Let us fix an arbitrary even $j=2 s, s \in \mathbb{N}$. By Lemma 1 , the function $\varphi$ has exactly $j$ zeros in the circle $\left\{x:|x|<\rho_{j}\right\}, \rho_{j}:=q_{2} q_{3} \cdot \ldots \cdot q_{j} \sqrt{q_{j+1}}$. Our aim is to show that all this zeros are 
real. We observe that $z_{0} \in\left[1, q_{2}\right], \rho_{2}=q_{2} \sqrt{q_{3}}>z_{0}, r_{3}=q_{2} q_{3} z_{0}>\rho_{2}, \rho_{4}=q_{2} q_{3} q_{4} \sqrt{q_{5}}>r_{3}$, $r_{5}=q_{2} q_{3} q_{4} q_{5} z_{0}>\rho_{4}, \ldots, r_{j-1}=q_{2} q_{3} \cdot \ldots \cdot q_{j-1} z_{0}>\rho_{j-2}, \rho_{j}:=q_{2} q_{3} \cdot \ldots \cdot q_{j} \sqrt{q_{j+1}}>r_{j-1}$ (see Lemma 2 and Lemma 3 for the definitions of $\rho_{j}$ and $r_{j}$ ). Finally, we get

$$
0<z_{0}<\rho_{2}<r_{3}<\rho_{4}<r_{5}<\ldots<r_{j-1}<\rho_{j},
$$

and, by Lemma 2 and Lemma 3 we have

$$
\begin{gathered}
\varphi(0)>0, \varphi\left(z_{0}\right) \leq 0, \varphi\left(\rho_{2}\right) \geq 0, \varphi\left(r_{3}\right) \leq 0, \varphi\left(\rho_{4}\right) \geq 0, \\
\varphi\left(r_{5}\right) \leq 0, \ldots, \varphi\left(\rho_{j-2}\right) \geq 0, \varphi\left(r_{j-1}\right) \leq 0 .
\end{gathered}
$$

Thus, we find at least $j-1$ real zeros of $\varphi$ in the circle $\left\{x:|x|<\rho_{j}\right\}$, and, since $\varphi$ has exactly $j$ zeros in this circle, all the zeros in the circle are real. By the fact that $\rho_{j} \rightarrow \infty$ for $j \rightarrow \infty$, we conclude that all the zeros of $\varphi$ are real.

\section{Proof of Theorem 2.}

Proof. During the proof we will consider the function $\varphi_{a, b}(z)=f_{a, b}(-z)$ instead of $f_{a, b}$.

If $\varphi_{a, b} \in \mathcal{L}-\mathcal{P}$, i.e. belongs to the Laguerre-Pólya class, then there exists $z_{0} \in\left[0, \frac{a_{1}}{a_{2}}\right]=$ $[0, a]$, such that $\varphi_{a, b}\left(z_{0}\right) \leq 0$.

As we mentioned in the proof of Lemma 3 , for $x \in[0,1]$ we have

whence $\varphi_{a, b}(x)>0$ for all $x \in[0,1]$.

$$
1 \geq x>\frac{x^{2}}{q_{2}}>\frac{x^{3}}{q_{2}^{2} q_{3}}>\frac{x^{4}}{q_{2}^{3} q_{3}^{2} q_{4}}>\cdots,
$$

Thus, we have $z_{0} \in(1, a]$. For any $x \in(1, a]$ we have

$$
\varphi_{a, b}(x)=1-x+\frac{x^{2}}{a}+\left(-\frac{x^{3}}{a^{2} b}+\frac{x^{4}}{a^{3} b^{2} a}\right)+\left(-\frac{x^{5}}{a^{4} b^{3} a^{2} b}+\frac{x^{6}}{a^{5} b^{4} a^{3} b^{2} a}\right)+\cdots
$$

We need the inequality: $\frac{\partial}{\partial b}\left(-\frac{x^{3}}{a^{2} b}+\frac{x^{4}}{a^{3} b^{2} a}\right)=\frac{x^{3}}{a^{2} b^{2}}-\frac{2 x^{4}}{a^{4} b^{3}} \geq 0$, which is equivalent to $x \leq \frac{a^{2} b}{2}$, that is correct for $x \in(1, a]$. Therefore, $\left(-\frac{x^{3}}{a^{2} b}+\frac{x^{4}}{a^{3} b^{2} a}\right)$ is increasing in $b$, which follows that since, under our assumptions, $a<b$.

$$
-\frac{x^{3}}{a^{2} b}+\frac{x^{4}}{a^{3} b^{2} a} \geq-\frac{x^{3}}{a^{3}}+\frac{x^{4}}{a^{6}}
$$

Analogously, for all $k \geq 2$

$$
\begin{gathered}
\frac{\partial}{\partial b}\left(-\frac{x^{2 k-1}}{a^{2 k-2} b^{2 k-3} \cdot \ldots \cdot a^{2} b}+\frac{x^{2 k}}{a^{2 k-1} b^{2 k-2} \cdot \ldots \cdot b^{2} a}\right)= \\
=\frac{\partial}{\partial b}\left(-\frac{x^{2 k-1}}{a^{(k-1) k} b^{(k-1)^{2}}}+\frac{x^{2 k}}{a^{k^{2}} b^{(k-1) k}}\right)=\frac{(k-1)^{2} \cdot x^{2 k-1}}{a^{(k-1) k} \cdot b^{(k-1)^{2}+1}}-\frac{(k-1) k \cdot x^{2 k}}{a^{k^{2}} \cdot b^{(k-1) k+1}} \geq 0,
\end{gathered}
$$

or, equivalently, $x \leq \frac{k-1}{k} a^{k} b^{k-1}$, which is correct for $x \in(1, a]$ and $k \geq 2$. Therefore,

$$
-\frac{x^{2 k-1}}{a^{(k-1) k} b^{(k-1)^{2}}}+\frac{x^{2 k}}{a^{k^{2}} b^{(k-1) k}} \geq-\frac{x^{2 k-1}}{a^{\frac{(2 k-1)(2 k-2)}{2}}}+\frac{x^{2 k}}{a^{\frac{2 k(2 k-1)}{2}}} .
$$

Thus, for any $x \in(1, a]$,

$$
\varphi_{a, b}(x)=1-x+\frac{x^{2}}{a}-\frac{x^{3}}{a^{2} b}+\frac{x^{4}}{a^{3} b^{2} a}-\cdots \geq 1-x+\frac{x^{2}}{a}-\frac{x^{3}}{a^{3}}+\frac{x^{4}}{a^{6}}-\cdots=g_{\sqrt{a}}(-\sqrt{a} x),
$$

where $g_{a}$ is the partial theta-function.

Since $\varphi_{a, b}\left(z_{0}\right) \leq 0$ for $z_{0} \in(1, a]$, we have $g_{\sqrt{a}}\left(-\sqrt{a} z_{0}\right) \leq 0$. Denote by $y_{0}:=-\sqrt{a} z_{0}$, then $y_{0} \in\left(\sqrt{a},(\sqrt{a})^{3}\right]$. So, for the partial theta-function $g_{\sqrt{a}}$ there exists a point $y_{0} \in\left(\sqrt{a},(\sqrt{a})^{3}\right]$ such that $g_{\sqrt{a}}\left(y_{0}\right) \leq 0$. By Theorem E, $g_{\sqrt{a}} \in \mathcal{L}-\mathcal{P}$, whence $(\sqrt{a})^{2} \geq q_{\infty}$. 
Thus, we have proved statement 1 of Theorem 2 .

Statement 2 follows from the reasoning above. Suppose that the numbers $a, b, q_{\infty} \leq a<b$, are such that $\varphi_{a, b} \in \mathcal{L}-\mathcal{P}$. By Theorem 1, there exists a point $z_{0} \in(1, a]$ such that $\varphi_{a, b}\left(z_{0}\right) \leq$ 0 . As we have proved below, for every fixed $a$ and $x \in(1, a]$, the expression for $\varphi_{a, b}$ is nondecreasing in $b$. Thus, if $a<c<b$ then $\varphi_{a, c}\left(z_{0}\right) \leq \varphi_{a, b}\left(z_{0}\right) \leq 0$. By Theorem $1, \varphi_{a, c} \in \mathcal{L}-\mathcal{P}$, and we have proved the statement 2 of Theorem 2 .

Statement 3 can be proved analogously. For every $x \in(1, a]$, we have

$$
\varphi_{a, b}(x)=1-x+\left(\frac{x^{2}}{a}-\frac{x^{3}}{a^{2} b}\right)+\left(\frac{x^{4}}{a^{3} b^{2} a}-\frac{x^{5}}{a^{4} b^{3} a^{2} b}\right)+\left(\frac{x^{6}}{a^{5} b^{4} a^{3} b^{2} a}-\frac{x^{7}}{a^{6} b^{5} a^{4} b^{3} a^{2} b}\right)+\cdots
$$

We need the inequality $\frac{\partial}{\partial a}\left(\frac{x^{2}}{a}-\frac{x^{3}}{a^{2} b}\right)=-\frac{x^{2}}{a^{2}}+\frac{2 x^{3}}{a^{3} b} \leq 0$, which is equivalent to $x \leq \frac{a b}{2}$, that is correct for $x \in(1, a]$. Therefore, $\left(\frac{x^{2}}{a}-\frac{x^{3}}{a^{2} b}\right)$ is decreasing in $a$.

Analogously, for all $k \geq 2$

$$
\begin{gathered}
\frac{\partial}{\partial a}\left(\frac{x^{2 k}}{a^{2 k-1} b^{2 k-2} \cdot \ldots \cdot b^{2} a}-\frac{x^{2 k+1}}{a^{2 k} b^{2 k-1} \cdot \ldots \cdot b^{3} a^{2} b}\right)=\frac{\partial}{\partial a}\left(\frac{x^{2 k}}{a^{k^{2}} b^{k(k-1)}}-\frac{x^{2 k+1}}{a^{k(k+1)} b^{k^{2}}}\right)= \\
=-\frac{k^{2} \cdot x^{2 k}}{a^{k^{2}+1} \cdot b^{k(k-1)}}+\frac{k(k+1) \cdot x^{2 k+1}}{a^{k^{2}+k+1} \cdot b^{k^{2}}} \leq 0,
\end{gathered}
$$

or, equivalently, $x \leq \frac{k}{k+1} a^{k} b^{k}$, which is correct for $x \in(1, a]$ and $k \geq 2$. Therefore, for every fixed $b, b>a$, and $x \in(1, a]$ the expression for $\varphi_{a, b}$ is non-increasing in $b$. Suppose that the numbers $a, b, q_{\infty} \leq a<b$, are such that $\varphi_{a, b} \in \mathcal{L}-\mathcal{P}$. By Theorem 1 , there exists a point $z_{0} \in(1, a]$ such that $\varphi_{a, b}\left(z_{0}\right) \leq 0$. Thus, if $a<d<b$ then $\varphi_{d, b}\left(z_{0}\right) \leq \varphi_{a, b}\left(z_{0}\right) \leq 0$ and $z_{0} \in(1, a] \subset(1, d]$. By Theorem 1, $\varphi_{d, b} \in \mathcal{L}-\mathcal{P}$, and we have proved the statement 3 of Theorem 2 .

Aknowledgement. The research was supported by the National Research Foundation of Ukraine funded by Ukrainian State budget in frames of project 2020.02/0096 "Operators in infinite-dimensional spaces: the interplay between geometry, algebra and topology".

\section{REFERENCES}

1. A. Bohdanov, Determining bounds on the balues of barameters for a bunction $\varphi_{a}(z, m)=\sum_{k=0}^{\infty} \frac{z^{k}}{a^{k^{2}}}(k !)^{m}$, $m \in(0,1)$, to belong to the Laguerre-Pólya class, Comput. Methods Funct. Theory, (2017), DOI:10.1007/ s40315-017-0210-6.

2. A. Bohdanov, A. Vishnyakova, On the conditions for entire functions related to the partial thetafunction to belong to the Laguerre-Pólya class, J. Math. Anal. Appl., 434 (2016), №2, 1740-1752. DOI:10.1016/j.jmaa.2015.09.084

3. T. Craven, G. Csordas, Complex zero decreasing sequences, Methods Appl. Anal., 2 (1995), $420-441$.

4. G. Gasper, M. Rahman, Basic hypergeometric series, Encyclopedia of mathematics and its applications, Cambridge University Press, United Kingdom, Cambridge, 2004.

5. I.I. Hirschman, D.V. Widder, The convolution transform, Princeton University Press, Princeton, New Jersey, 1955.

6. J. I. Hutchinson, On a remarkable class of entire functions, Trans. Amer. Math. Soc., 25 (1923), 325-332.

7. O. Katkova, T. Lobova and A. Vishnyakova, On power series having sections with only real zeros, Comput. Methods Funct. Theory, 3 (2003), №2, 425-441.

8. O. Katkova, T. Lobova, A. Vishnyakova, On entire functions having Taylor sections with only real zeros, J. Math. Phys., Anal., Geom., 11, (2004), №4, 449-469. 
9. V.P. Kostov, About a partial theta function, C. R. Acad. Bulgare Sci., 66, (2013), 629-634.

10. V.P. Kostov, On the zeros of a partial theta function, Bull. Sci. Math., 137 (2013), №8, 1018-1030.

11. V.P. Kostov, On the spectrum of a partial theta function, Proc. Roy. Soc. Edinburgh Sect. A, 144 (2014), №05, 925-933.

12. V.P. Kostov, Asymptotics of the spectrum of partial theta function, Revista Matematica Complutense, 27 (2014), №2, 677-684.

13. V.P. Kostov, A property of a partial theta function, C. R. Acad. Bulgare Sci., 67 (2014), 1319-1326.

14. V.P. Kostov, Asymptotic expansions of zeros of a partial theta function, C. R. Acad. Bulgare Sci., 68 (2015), 419-426.

15. V.P. Kostov, On the double zeros of a partial theta function, Bulletin des Sciences Mathamatiques, 140 (2016), №4, 98-111.

16. V.P. Kostov, On a partial theta function and its spectrum, Proceedings of the Royal Society of Edinburgh Section A: Mathematics, 146 (2016), №3, 609-623.

17. V.P. Kostov, The closest to 0 spectral number of the partial theta function, C. R. Acad. Bulgare Sci., 69, (2016), 1105-1112.

18. V.P. Kostov, B. Shapiro, Hardy-Petrovitch-Hutchinson's problem and partial theta function, Duke Math. J., 162 (2013), №5, 825-861.

19. B.Ja. Levin, Distribution of Zeros of Entire Functions, Transl. Math. Mono., 5, Amer. Math. Soc., Providence, RI, 1964; revised ed. 1980.

20. T.H. Nguyen, A. Vishnyakova, On the entire functions from the Laguerre-Pólya class having the decreasing second quotients of Taylor coefficients, Journal of Mathematical Analysis and Applications, 465 (2018), №1, 348-359. https://doi .org/10.1016/j · jmaa.2018.05.018.

21. T.H. Nguyen, A. Vishnyakova, On the necessary condition for entire function with the increasing second quotients of Taylor coefficients to belong to the Laguerre-Pólya class, Journal of Mathematical Analysis and Applications, 480 (2019), №2. https://doi.org/10.1016/j.jmaa.2019.123433.

22. T.H. Nguyen, A. Vishnyakova, On the closest to zero roots and the second quotients of Taylor coefficients of entire functions from the Laguerre-Pólya I class, Results in Mathematics, 75 (2020), №115. https: //doi.org/10.1007/s00025-020-01245-w.

23. T.H. Nguyen, A. Vishnyakova, On the entire functions from the Laguerre-Pólya I class having the increasing second quotients of Taylor coefficients, Journal of Mathematical Analysis and Applications, 498 (2021), №1. https ://doi .org/10 .1016/j . jmaa . 2021 . 124955.

24. T.H. Nguyen, A. Vishnyakova, On the number of real zeros of real entire functions with a non-decreasing sequence of the second quotients of Taylor coefficients. https://arxiv.org/abs/2101.11757

25. T.H. Nguyen, On the conditions for a special entire function related to the partial theta-function and the Euler function to belong to the Laguerre-Pólya class, Computational Methods and Function Theory, (2021). https://doi.org/10.1007/s40315-021-00361-0

26. N. Obreschkov, Verteilung und Berechnung der Nullstellen reeller Polynome, VEB Deutscher Verlag der Wissenschaften, Berlin, 1963.

27. G. Pólya, Collected Papers, V.II Location of Zeros, (R.P.Boas ed.) MIT Press, Cambridge, MA, 1974.

28. G. Pólya, J. Schur, Über zwei Arten von Faktorenfolgen in der Theorie der algebraischen Gleichungen, J. Reine Angew. Math., 144 (1914), 89-113.

29. A.D. Sokal, The leading root of the partial theta function, Advances in Mathematics, 229 (2012), №5, 2063-2621.

30. S. O. Warnaar, Partial theta functions.

https://www.researchgate.net/publication/327791878_Partial_theta_functions

Department of Mathematics \& Computer Sciences

V.N. Karazin Kharkiv National University

Kharkiv, Ukraine

nguyen.hisha@karazin.ua

anna.vishnyakova@karazin.ua 